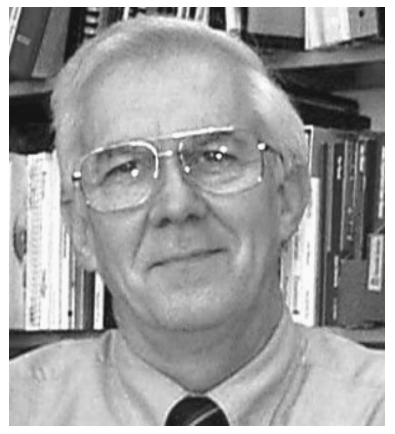

\section{Engineering a New Society}

In the fields of science and engineering you have to deal with what you have or what you can fabricate. Any mathematical study that does not have some physical relevance is useless except as an object of calculational art-to be appreciated, but not used. As G. H. Hardy noted in $A$ Mathematician's Apology, “ 'Imaginary' universes are so much more beautiful than this stupidly constructed 'real' one; and most of the finest products of an applied mathematician's fancy must be rejected, as soon as they have been created, for the brutal but sufficient reason that they do not fit the facts.' The principle in this mathematics example applies to the serious feasibility design study being done to combine OSA and SPIE into a unified society. When trying to combine these two strong, vigorous societies in the field of optics, you cannot make the new organization as streamlined as you might want. You have to deal with the history and current status of our societies. You have work with what's available.

At their meetings in October, the Boards of Directors of SPIE and OSA asked the Joint Task Force (JTF) on closer collaboration between the societies to continue pursuit of a merger of the two societies. It also released the full report of the Task Force on the SPIE and OSA web pages so that the members of the two societies could examine the rationale and the details of the new organization. I urge you to read at least the Executive Summary and to examine those parts of the full report that are of interest to you. In addition, there will be an electronic bulletin board so that you can post comments and carry on threaded dialogs on the merger and on details of its implementation.

The JTF, for those who have not yet read the Executive Summary or the full report, has recommended "a union of OSA and SPIE that is simultaneously a merger of operations and a federation of cultures.' In arriving at this organizational structure the JTF examined a range of possible arrangements, but this was the only one that permitted the collaboration of common efforts, such as membership, education, public policy, meeting management (but not meeting organization), and publication production (but not publication policies). At the same time the pro- posed structure preserves the OSA and SPIE cultures where meetings are planned and publication policies are set. It also allows individuals and technical groups that wish to remain affiliated with their original societies and to permit those groups that can establish separate identities to become full-fledged divisions of the new organization. In that way this optics organization becomes extensible and responsive to the needs of new fields within optics. It is a good system design.

This structure of the new organization grew out of the studies and discussions that were carried out by the Task Force during its six-month investigation. The approach of the JTF was cautious. The attitude, as I interpreted it in the meetings, was that we have two successful societies and any move toward unity had to deal with their current status. The new organizational design had to fit the facts. At the beginning of the proceedings it certainly was not obvious to anyone participating in the JTF that this merger/federation concept would be the result of its work.

What was clear from the very beginning was that " "preservation of the cultures" of the two societies was a major goal. Recently I have seen comments stating that the new society should be rejected by the members not because it goes too far, but because it doesn't go far enough. "Just merge, dammit!"' is the short version of these responses. But a closer look at the way that meetings are organized and information is transmitted would give pause to anyone contemplating instant amalgamation of these societies. The engineering has to be done through a realistic evaluation of the current resources and policies.

One of the major benefits of this new structure is that it allows the two founding societies to "step out" together, maintaining practices and alliances that are already in place as the new society is established. When conditions change, as they do rapidly in optics, the two divisions can take advantage of their strengths and combine their interests and expertise in new ventures. Without the new structure, they would be doomed to unproductive competition in new fields that both could claim. It is this waste that this new design can address in an adiabatic manner.

A number of past presidents of SPIE have weighed in against the merger. Several have described the new organization as "another layer of bureaucracy.' One felt that this was a ploy by OSA to use SPIE to reinvigorate a 
static organization. Another felt the "fringe" applications that SPIE welcomes would be cast out by the OSA once the organization is completed. As one past president put it: "The OSA is thoroughly dominated by an academic clique which despises and looks down on SPIE." Another said that the Operations section of the report was "lacking in any significant detail.,"

I cannot deal with all of these objections here, but I would like to make a few observations based on my months of working on the JTF and co-chairing the Meetings and Exhibits subcommittee. The "layer of bureaucracy" charge is a bit hard to pin down. Based on our study the staffs serving the current societies will not change much. They are operating efficiently, providing good service to members, and keeping both of the societies in the black. In the case of governance the number of committees should change very little because some of the common committees will be consolidated at the top level of the organization. There may be additional opportunities for members to get involved with the governance of the organization and I think that is all to the good. As for the prospects of SPIE being taken over by academics, there are fears by some of my friends in OSA that their topical meetings will eliminated by the engineers or they will be forced to hold them in upstairs conference rooms in Madison Square Garden during New York Knicks games. I have heard similar fears expressed by some SPIE members. My own sense of the governance of the new society is that those in leadership positions will be sensitive to the needs of the various segments of our society or they will lose them to another organization. The current discussion that is being organized by the leadership of both societies is an example of that. Although I understand many of their objections, I cannot count them as providing a barrier to the decision to create a new organization. Regarding the charge of an incomplete operational plan, I credit that fully. It was the charge of the Boards to determine feasibility and there are now the resolutions by the Boards that their individual societies continue the process and do "due diligence" in structuring the combined operations. There are additional design details to be worked out.

For individual members there are substantial benefits to be had if the merger goes through. We will have a single organization to belong to and our benefits will be greater than they are today for either organization. Those of us who belong to both organizations will benefit by significant savings in membership dues. In addition to member rates on meetings and publications, the range of topics and areas covered by the two organizations will provide access to more information. Because our professional efforts are based on access to the widest range and most up-to-date information in our field, a combined database of technical information is a definite benefit of a merger. The separate organizations, OSA and SPIE, have served the optical scientist and optical engineer for the greater part of this century. And have done it well. This new organization is intended to do the same for optics in the next century.

Since 1996 all of the articles published in this journal are available not only on paper, but as "digital ink." That is, the text and pictures that constitute the articles are photocomposed and stored as electronic files. The files are printed with other auxiliary copy, such as the table of contents. But the electronic files used in this process are now available for further search and linking to other parts of this new landscape of information that is being built by our societies. Without a merger, a system of tariffs and external search engines would be necessary to connect and access these resources. Anyone who has dealt with generalized search engines knows the frustration of sifting through the chaff of irrelevant choices. With a merger, these two societies, one publishing the largest peerreviewed optics literature in the world, the other publishing the most up-to-date set of conference proceedings in the field of optics, will establish for the benefit of their members and the optics community a new interconnected optics resource. No other two entities can do it better and neither can do it alone.

We've got a good system design. And a good system design permits one to do what good engineering should do: address the details needed to make things work.
Donald C. O'Shea Editor 